\title{
Erratum to: Expression and characterization of two new alkane-inducible cytochrome P450s from Trichoderma harzianum
}

R. Del Carratore · P. G. Gervasi - M. P. Contini •

P. Beffy - B. E. Maserti - G. Giovannetti •

A. Brondolo $\cdot$ V. Longo

Published online: 17 March 2011

(C) Springer Science+Business Media B.V. 2011

\section{Erratum to: Biotechnol Lett DOI 10.1007/s10529-011-0557-0}

Unfortunately, first author's family name and given names had been wrongly published. The correct version is First name: " $R$ " and the Last name "Del Carratore".

The online version of the original article can be found under doi:10.1007/s10529-011-0557-0.

R. Del Carratore $(\bowtie) \cdot$ P. G. Gervasi ·

M. P. Contini · P. Beffy

Institute of Clinical Physiology, National Research

Council (CNR), via Moruzzi 1, Pisa, Italy

e-mail: rdc@ifc.cnr.it

V. Longo

Institute of Agricultural Biology and Biotechnology,

CNR, via Moruzzi 1, Pisa, Italy

B. E. Maserti

Institute of Biophysics, CNR, via Moruzzi 1, Pisa, Italy

G. Giovannetti · A. Brondolo

Experimental Culture Centre, Valle D'Aosta,

Fraz. Olleyes 9, Quart, AO, Italy 